\title{
Mmu_circ_003795 regulates osteoblast differentiation and mineralization in MC3T3-E1 and MDPC23 by targeting COL15A1
}

\author{
JINGWEN WU $^{1 *}$, WEN REN ${ }^{1 *}$, ZHICHAO ZHENG $^{1}$, ZHU HUANG $^{1}$, TINGTING LIANG $^{1}$, FUYAO LI $^{1}$, \\ ZHAN SHI ${ }^{2}$, QIANZHOU JIANG ${ }^{1}$, XUECHAO YANG ${ }^{1}$ and LVHUA GUO $^{1}$ \\ ${ }^{1}$ Key Laboratory of Oral Medicine, Guangzhou Institute of Oral Disease, Stomatology Hospital of \\ Guangzhou Medical University, Guangzhou, Guangdong 510140, P.R. China; ${ }^{2}$ Faculty of Arts and Science, \\ Human Biology Program, University of Toronto, Toronto, Ontario M5S 1A1, Canada
}

Received August 12, 2019; Accepted April 28, 2020

DOI: $10.3892 / \mathrm{mmr} .2020 .11264$

\begin{abstract}
Circular RNAs (circRNAs) are a class of non-coding RNAs that exhibit important regulatory roles in various biological processes. However, the role of circRNAs and their potential role in osteoblast differentiation and mineralization is unclear. The aim of the present study was to investigate the expression of mmu_circ_003795 and its effect on collagen type XV $\alpha 1$ chain (COL15A1). First, it was identified that the expression levels of mmu_circ_003795 and osteopontin (OPN) were upregulated in the induced cells. Silencing of $\mathrm{mmu}_{-}$ circ_003795 reduced the gene and protein levels of COL15A1 and OPN, whereas the expression level of mmu-microRNA (miR)-1249-5p was upregulated. In addition, after 7 or 14 days of induction, alkaline phosphatase and Alizarin Red-S staining were decreased in the mmu_circRNA_003795 inhibitory group compared with the negative control group. In conclusion, mmu_circ_003795 may regulate osteoblast differentiation and mineralization in MC3T3-E1 and MDPC23 cells via mmu-miR-1249-5p by targeting COL15A1.
\end{abstract}

\section{Introduction}

Non-healing bone defects due to trauma, infection and tumor ablation restrict the development of oral implant restoration (1).

Correspondence to: Professor Lvhua Guo or Professor Qianzhou Jiang, Key Laboratory of Oral Medicine, Guangzhou Institute of Oral Disease, Stomatology Hospital of Guangzhou Medical University, 39 Huangsha Road, Guangzhou, Guangdong 510140, P.R. China

E-mail: fsglh1@163.com

E-mail: jqianzhou@126.com

*Contributed equally

Key words: circRNA, osteopontin, collagen type XV $\alpha 1$ chain, osteoblast differentiation, mineralization
Therefore, identifying ways to accelerate bone integration has become an urgent requirement. Due to their strong osteogenesis, ease of culture and availability, MC3T3-E1 and MDPC23 cells are considered good candidates for alveolar bone regeneration. Osteogenesis is a complex process that is strictly regulated by numerous pathways (2). The molecular mechanism of osteogenesis and mineralization remains unclear.

Circular RNAs (circRNAs) are a new class of non-coding RNAs that serve important roles in multiple biological processes $(3,4)$. Due to advances in science and technology, particularly in bioinformatics and RNA sequencing technologies, it has been determined that numerous exon transcripts can be used to form circRNAs by nonlinear reverse splicing or gene rearrangement. circRNA molecules that exhibit a closed ring structure are thought to serve an important role in bone metabolism $(5,6)$. For example, osteogenesis can be enhanced by mm9_circ_009056, which upregulates bone morphogenetic protein 7 by targeting microRNA (miR/miRNA)-22-3p (7). Additionally, the circRNA CDR1-AS can activate Smad1/5/8 and p38 mitogen associated protein kinase pathways to promote osteogenesis of periodontal ligament stem cells by inhibiting miR-7 expression (8). Therefore, the present study attempted to explain the molecular mechanism of osteogenesis and mineralization via circRNAs.

The authors' previous study found that mmu_circ_003795 was significantly upregulated in bone marrow-derived stem cells (BMSCs) undergoing cell proliferation, demonstrating that mmu_circ_003795 may serve an important role in the proliferation of BMSCs (9). The present study further investigated the function of mmu_circ_003795 in the osteogenesis of MC3T3-E1 and MDPC23 cells.

\section{Materials and methods}

Cell culture. MC3T3-E1 (American Type Culture Collection) and MDPC23 (Cellcook) cells were plated on 6-well plate culture dishes at density of $1 \times 10^{5}$ cells/well. In addition, $2 \mathrm{ml}$ Dulbecco's modified Eagle's medium (Gibco; Thermo Fisher Scientific, Inc.) containing $10 \%$ fetal bovine serum (Gibco; Thermo Fisher Scientific, Inc.) was added to each well. Cells were maintained in a humidified atmosphere of $95 \%$ air and $5 \% \mathrm{CO}_{2}$ at $37^{\circ} \mathrm{C}$. 
Transfection of small interfering RNAs (siRNAs), mimics and inhibitors. The siRNAs, mimics and inhibitors were designed and synthesized by Guangzhou RiboBio Co., Ltd. (siRNA: Sense: 5'-GCUAGAACAGCAUGGUCCAdT dT-3', antisense: 3'-dTdTCGAUCUUGUCGUACCAGGU-5'; inhibitors: 5'-UCCUCCCUCCCCUACCCGGUUCAAG-3'; mimic sense: 5'-AGGAGGGAGGGGAUGGGCCAAGUU C-3', antisense: 5'-UCCUCCCUCCCCUACCCGGUUCAA G-3') (Table I). MC3T3-E1 and MDPC23 cells were seeded in 6-well plates at a density of $1 \times 10^{5} /$ well. The cells were transfected with $20 \mathrm{nM}$ siRNA mimics or inhibitors using GenMute $^{\mathrm{TM}}$ siRNA Transfection kit and Transfection Buffer (SignaGen Laboratories) according to the manufacturer's protocol. To ensure a high silencing efficiency, the transfection medium with siRNAs was changed every $72 \mathrm{~h}$. Experiments were performed 24-72 $\mathrm{h}$ post transfection.

Microarray hybridization. The MDPC23 cells were divided into two groups and treated as before. In the subject group, $1 \times 10^{5}$ cells were treated with the induction medium containing $20 \mathrm{nM}$ siRNA of mmu_circ_003795 for 7 days. In the control groups, the cells $\left(1 \times 10^{5}\right)$ were treated with the induction medium containing $20 \mathrm{nM}$ negative control siRNA for 7 days. The sequences of siRNA were as follows: (Sense 5'-GCUAGA ACAGCAUGGUCCAdTdT-3' and antisense 3'-dTdTCGAUC UUGUCGUACCAGGU-5'). These two groups were used to detect the expression of mRNAs and miRNAs following silencing of mmu_circRNA_003795. Total RNA from each sample was quantified using the NanoDrop ND-2000 (Thermo Fisher Scientific, Inc.). The sample preparation and microarray hybridization were performed based on the standard protocols of the BGI group (10).

Detection of alkaline phosphatase (ALP) activity. Cells were seeded into 48 -well plates at a density of $5 \times 10^{3}$ cells/well. The MDPC23 and MC3T3-E1 cells were divided into two groups: The control group and the subject group. In the subject group, the cells $\left(5 \times 10^{3}\right)$ were treated with the induction medium containing $20 \mathrm{~nm}$ siRNA of mmu_circ_003795 for 14 days. In the control group, the cells $\left(5 \times 10^{3}\right)$ were treated with the induction medium containing $20 \mathrm{nM}$ negative control siRNA for 14 days. The medium was changed every 3 days. An Alkaline Phosphatase Staining Kit (BestBio, Inc.) was used to detect the ALP activity, according to the manufacturer's protocol.

Alizarin Red-S (ARS) staining. Mineralization in MC3T3-E1 and MDPC 23 cells was measured by staining with ARS. Cells were seeded in 48 -well plates at a density of $5 \times 10^{3}$ cells/well. As previously the MDPC23 and MC3T3-E1 cells were divided into two groups. In the subject group, the cells were treated with the induction medium containing $20 \mathrm{~nm}$ siRNA of mmu_circ_003795. In the control group, the cells were treated with the induction medium containing $20 \mathrm{nM}$ negative control siRNA. The medium was changed every 3 days. Following culture for 21 days, cells were fixed with $4 \%$ paraformaldehyde for 10 min after washing three times with PBS at room temperature. Subsequently, the ARS solution (BestBio, Inc.) was used to stain the cells at $37^{\circ} \mathrm{C}$ for $30 \mathrm{~min}$. The stained cells were observed by a stereo light microscope (Leica Microsystems $\mathrm{GmbH}$ ). For quantification analysis, $10 \%$ hexadecyl pyridinium chloride monohydrate (CPC; Sigma-Aldrich; Merck KGaA) was used to dissolve the mineralized nodules and then the absorbance was measured at $562 \mathrm{~nm}$ using a Multiskan ${ }^{\mathrm{TM}}$ FC spectrophotometer (Thermo Fisher Scientific, Inc.).

Reverse transcription-quantitative polymerase chain reaction (RT-qPCR) and nucleic acid electrophoresis. The total RNA of BMSCs was extracted using TRIzol reagent (Thermo Fisher Scientific, Inc.). The quantity and quality of RNA were determined using a NanoDrop 2000. Following RNA extraction, SuperScript III reverse transcriptase (Invitrogen; Thermo Fisher Scientific, Inc.) was used to synthesize complementary DNA (cDNA), according to the manufacturer's protocol. In the reverse transcription reaction the temperature protocol was as follows: $65^{\circ} \mathrm{C}$ for $5 \mathrm{~min}$ and $4^{\circ} \mathrm{C}$ for $1 \mathrm{~min}$ in the first step, followed by $37^{\circ} \mathrm{C}$ for $1 \mathrm{~min}, 50^{\circ} \mathrm{C}$ for $60 \mathrm{~min}$ and $70^{\circ} \mathrm{C}$ for $15 \mathrm{~min}$ in the second step. Subsequently, qPCR was performed using $12.5 \mu \mathrm{l}$ SYBR Premix EX Taq (Takara Bio, Inc.), $0.5 \mu 1$ forward primer (10 pmol/l), $0.5 \mu 1$ reverse primer (10 pmol/li), $1 \mu \mathrm{l} \mathrm{cDNA}$ and $\mathrm{ddH}_{2} \mathrm{O}$ to create a final volume of $25 \mu \mathrm{l}$. The primers used are listed in Table I. The reaction conditions were as follows: $95^{\circ} \mathrm{C}$ for $30 \mathrm{sec}$; and $95^{\circ} \mathrm{C}$ for $5 \mathrm{sec}, 55^{\circ} \mathrm{C}$ for $30 \mathrm{sec}$ and $72^{\circ} \mathrm{C}$ for $30 \mathrm{sec}$ for 40 cycles. The instrument automatically generated the cycle quantification $(\mathrm{Cq})$ value of mRNA and the internal reference gene GAPDH. Cq value difference $(\Delta \mathrm{Cq})$ indicated the relative expression of each mRNA investigated (11).

Identification of differentially expressed mRNAs. Quantile normalization of the original data and subsequent data processing was performed using the $\mathrm{R}$ software package (version 3.1.2) (12). After quantile normalization of the original data was performed, low-intensity filtering was carried out and the mRNAs with P (present) or M (marginal) ('all target values') tagged in at least one or two samples were retained for further analysis. When comparing the two sets of contours, the 'fold-change' (namely, the ratio of the group mean) between groups of mRNAs was calculated. The statistical significance of the differences between groups was determined using a t-test. mRNAs exhibiting a significant difference in expression exhibited a fold-change $>2$ and $\mathrm{P}<0.05$. The sorting and filtering function of Microsoft Excel 2019 (Microsoft Corporation) was used to filter data, analyze output and sort the mRNAs with differential expression according to the fold-changes and P-values. In addition, gene ontology (GO) analysis was performed to classify the related mRNAs (13). miRDB (http://www.mirdb.org/), TargetScan (http://www. targetscan.org/) and mirbases (http://www.mirbase.org/) were used to determine the corresponding target genes. All differentially expressed miRNAs were annotated in detail with information on the interaction between mmu_circ_003795 and miRNAs. Cytoscape (version 3.6.0; https://cytoscape.org) software was used to generate a network map.

Western blotting. Radioimmunoprecipitation assay buffer [50 mM Tris $\mathrm{HCl}$ (pH 7.4), $150 \mathrm{mM} \mathrm{NaCl}, 1 \%$ Nonidet P40 and $0.1 \%$ sodium dodecyl sulfate] and phenylmethanesulfonyl fluoride were used at $4^{\circ} \mathrm{C}$ to extract protein from the cells according to the standard procedure. The concentration was determined using a bicinchoninic acid protein assay kit (BestBio, Inc.). Total protein $(10 \mu \mathrm{g} / \mathrm{well})$ was separated using $10 \%$ SDS-PAGE and then 
Table I. The primers of the detected genes.

Name

Primer

COL15A1

ALP

OCN

GAPDH

OSX

RUNX2

mmu_circRNA_003795

mmu-miR-1249-5p

U6

Inhibitor-miR-1249-5p

Mimic-miR-1249-5p
F: 5'-CTCGCGGGTTACATAAGGCT-3'

R: 5'-GTAGAGGATAACCCGCTGGC-3'

F: 5'-GAGGCATACGCCATCACATG-3'

R: 5'-CCGATGGCACACCTGCTT-3'

F: 5'-TCTGACAAAGCCTTCATGTCCA-3'

R: 5'-AACGGTGGTGCCATAGAT-3'

F: 5'-AAGAAGGTGGTGAAGCAGG-3'

R: 5'-GAAGGTGGAAGAGTGGGAG-3'

F: 5'-GCAAATGACTACCCACCCTT-3'

R: 5'-ACGAGCCATAGGGATGAGTC-3'

F: 5'-ACTTGTGGCTGTTGTGATG-3'

R: 5'-TTGCTGTTGCTGTTGTTG-3'

F: 5'-CCTTAGCACCTGCCTTCTTG-3'

R: 5'-AGTTCACCCACTGTGCCTCC-3'

5'-AGGAGGGAGGGGATGGGCCAAGTTC-3'

5'-GCGCGTCGTGAAGCGTTC-3'

5'-UCCUCCCUCCCCUACCCGGUUCAAG-3'

F: 5'-AGGAGGGAGGGGAUGGGCCAAGUUC-3'

R: 5'-UCCUCCCUCCCCUACCCGGUUCAAG-3'

miR, microRNA; ALP, alkaline phosphatase; OCN, osteocalcin; RUNX2, runt-related transcription factor 2; OSX, Sp7 transcription factor; COL15A1, collagen type XV $\alpha 1$ chain; OPN, osteopontin; circ, circular; F, forward; R, reverse.

transferred to a polyvinylidene difluoride membrane. Skimmed milk was used to block membranes for $1 \mathrm{~h}$ at room temperature, then the blots were incubated with primary antibodies against OPN (1:1,000; catalog no. ab124830; Abcam), COL15A1 (1:1,000; catalog no. PA5-69518; Thermo Fisher Scientific, Inc.) and $\beta$-actin (1:5,000; catalog no. ab8226; Abcam) overnight at $4^{\circ} \mathrm{C}$. Following washing with $0.1 \%$ Tween PBS-T, the blots were incubated with goat anti-mouse horseradish peroxidase-conjugated secondary antibody (1:5,000; catalog no. ab6799; Abcam) for $1 \mathrm{~h}$ at room temperature. An enhanced chemiluminescent (ECL) kit (Beijing Dingguo Changsheng Biotechnology Co., Ltd.) was used to detect the proteins. ImageJ (version k1.45; National Institutes of Health) was used to measure densitometry.

Statistical analysis. All data were analyzed using the SPSS 11.5 software package (SPSS, Inc.). The data were expressed as the mean \pm standard deviation and the comparison of the data between groups was performed using paired t-test or two-way analysis of variance according to the nature of the data. Each experiment was repeated three times. The data of three groups were evaluated using analysis of variance and followed by Fisher's least significant difference post hoc test when the variance was normal, otherwise Dunnett's T3 test was used. $\mathrm{P}<0.05$ was considered to indicate a statistically significant difference.

\section{Results}

mmu_circ_003795 is upregulated during osteoblast differentiation of MC3T3-E1 and MDPC23 cells. MC3T3-E1 and MDPC23 cells were cultured with induction medium for
3 days. RT-qPCR was used to analyze the expression levels of mmu_circRNA_003795 and the osteogenic marker OPN, ALP, Sp7 transcription factor (OSX), osteocalcin (OCN) and runt-related transcription factor 2 (RUNX2). The results demonstrated that mmu_circRNA_003795, OPN, ALP, OSX, OCN and RUNX2 were increased (Fig. 1A). The ALP and ARS staining were performed to verify the successful induction (Fig. 1B and C). An siRNA sequence was designed to silence mmu_circRNA_003795. MDPC23 cells were transfected with negative control RNAs or siRNAs for 7 days. In the miRNA microarray, 1,729 miRNAs were different between the control and subject groups (Fig. 2A). The present study identified that mmu_circRNA_003795 is most likely bound to mmu-miR-1249-5p, mmu-miR-504-3p, mmu-miR-6399, mmu-miR-7054-5p and mmu-miR-667-5p (Fig. 2B). By comparing the aforementioned data, the present study selected mmu-miR-1249-5p as the target miRNA. The seed sequences of the circRNA were compared with miRNA sequences to identify the miRNAs that potentially bind to circRNAs (Fig. 2C).

Identification of differentially expressed $m R N A s$. GO analysis was performed to classify the related mRNAs. The analysis focused on 'Biological Process', 'Cellular Component' and 'Molecular Function' terms that may be associated with osteogenesis differentiation. The top GO terms were ranked by number of genes (Fig. 2D). miRDB (http://www. mirdb.org/), TargetScan (http://www.targetscan.org/) and mirbases (http://www.mirbase.org/) were used to determine the corresponding target genes of miR1249-5p. The above data was compared with the current mRNA microarray data. 
A

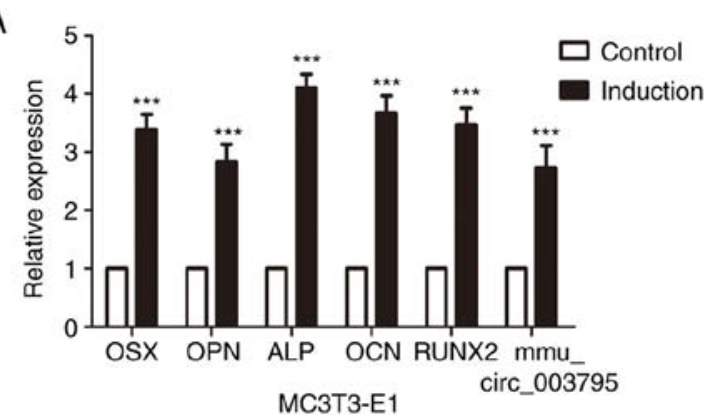

B

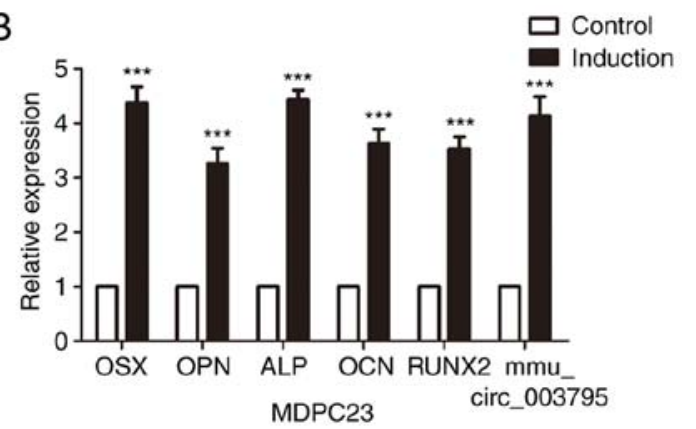

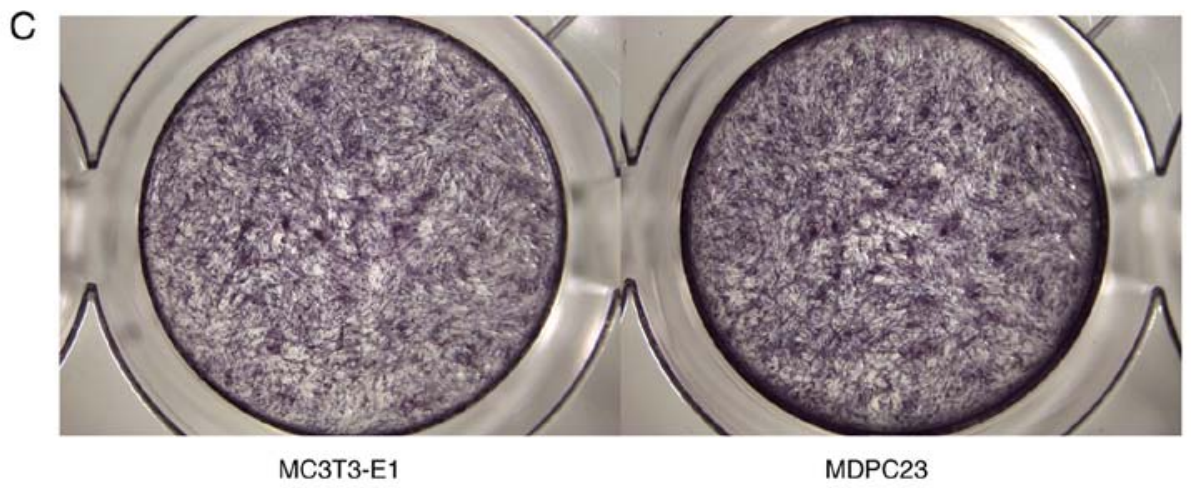

D

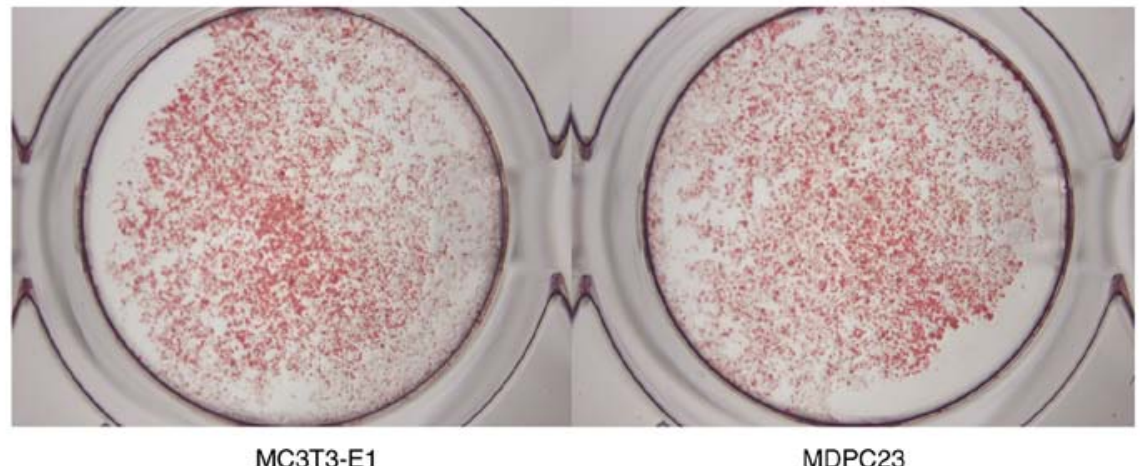

Figure 1. Expression of mmu_circRNA_003795 and OPN in MC3T3-E1 and MDPC23 cells. (A) Reverse transcription-quantitative PCR analysis of mmu_circRNA_003795 and OPN in MC3T3-E1 and MDPC23 cells following osteogenic induction. (B) The ALP assay of MC3T3-E1 and MDPC23 cells 10 days after induction. (C and D) The ARS assay of MC3T3-E1 and MDPC23 cells 21 days after induction. ${ }^{* * *} \mathrm{P}<0.005$ vs. the control. ALP, alkaline phosphatase; ARS, Alizarin Red-S; OPN, osteopontin; RUNX2, runt-related transcription factor 2; OSX, Sp7 transcription factor; circ, circular; OCN, osteocalcin.

According to the analysis, miR1249-5p and COL15A1 may have regulated osteoblast differentiation and mineralization in MDPC23 cells.

Alterations in the expression of miR1249-5p, COL15A1 and OPN following $\mathrm{mmu}$ _circRNA_003795 interference. siRNA was used to knockdown mmu_circRNA_003795 in MC3T3-E1 and MDPC23 cells. Compared with the negative control group, the expression of mmu_circRNA_003795 in the siRNA group was decreased in both MC3T3-E1 and MDPC23 cells (Fig. 3A and B). Following successful silencing of mmu_circRNA_003795, the expression of miR-1249-5p was increased both in MC3T3-E1 and MDPC23 cells (Fig. 3C and D). Compared with the negative control group, the expression of OPN was significantly decreased in the mRNA microarray. It is known that OPN is one of the early marker proteins involved in osteogenic differentiation (14). The present study speculates that OPN may be involved in regulating osteoblast differentiation and mineralization in MDPC 23 cells.
Therefore, the current study also tried to verify the changes in OPN when interfering with circ003795 and miR-1249-5p. The expression levels of COL15A1 and OPN were significantly decreased in both cell lines (Fig. 3E and F). Western blotting revealed the same result; the western blot analysis demonstrated that silencing of mmu_circRNA_003795 reduced the protein expression levels of COL15A1 and OPN (Fig. 3G and H).

Alterations in the expression of COL15A1 and OPN following miR1249-5p interference. An inhibitor and mimic were used to knockdown and overexpress miR1249-5p, respectively, in MC3T3-E1 and MDPC23 cells. Compared with the negative control group, the expression of miR1249-5p was decreased in the inhibitor group and increased in the mimic group in both MC3T3-E1 and MDPC23 cells (Fig. 4A and B). Following successful knockdown of miR1249-5p, the expression levels of COL15A1 and OPN were increased in MC3T3-E1 cells, whereas the expression levels of COL15A1 and OPN were decreased when miR1249-5p was overexpressed (Fig. 4C). 

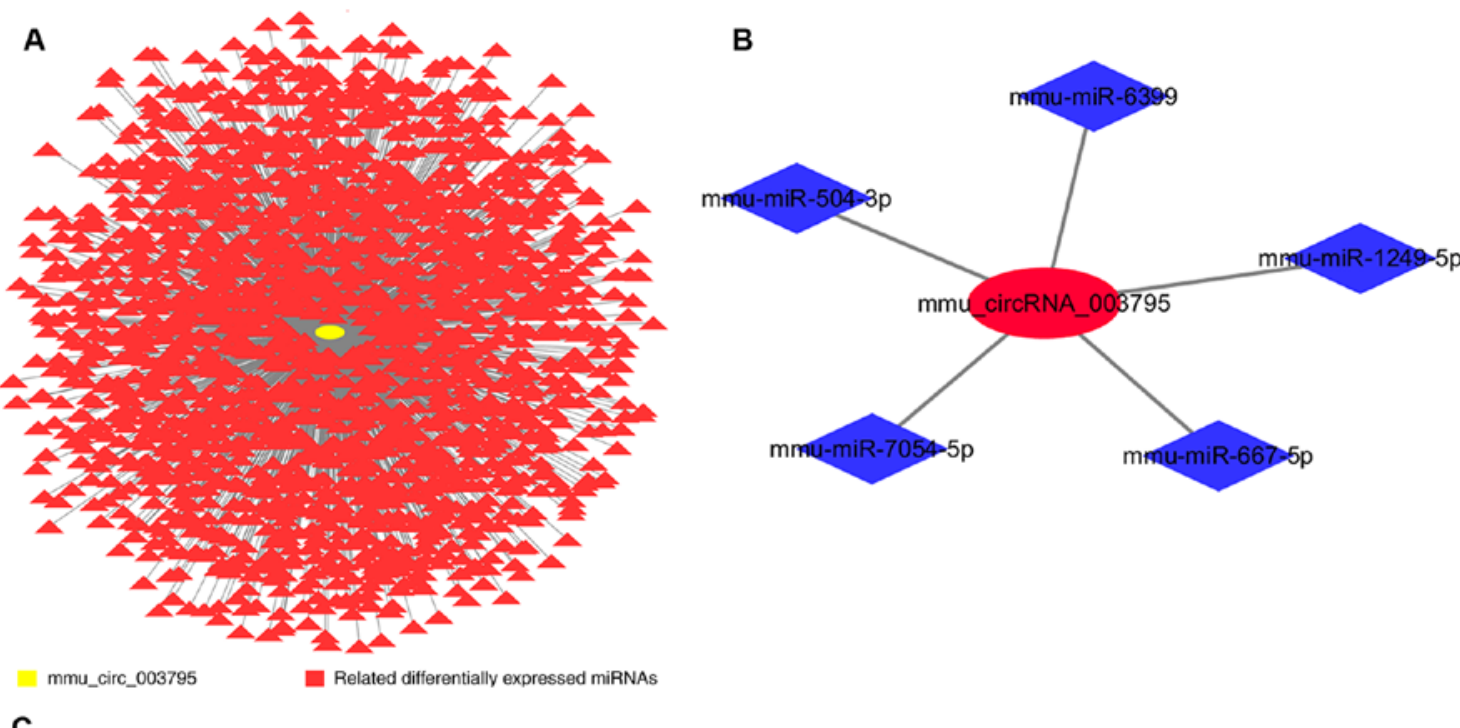

C

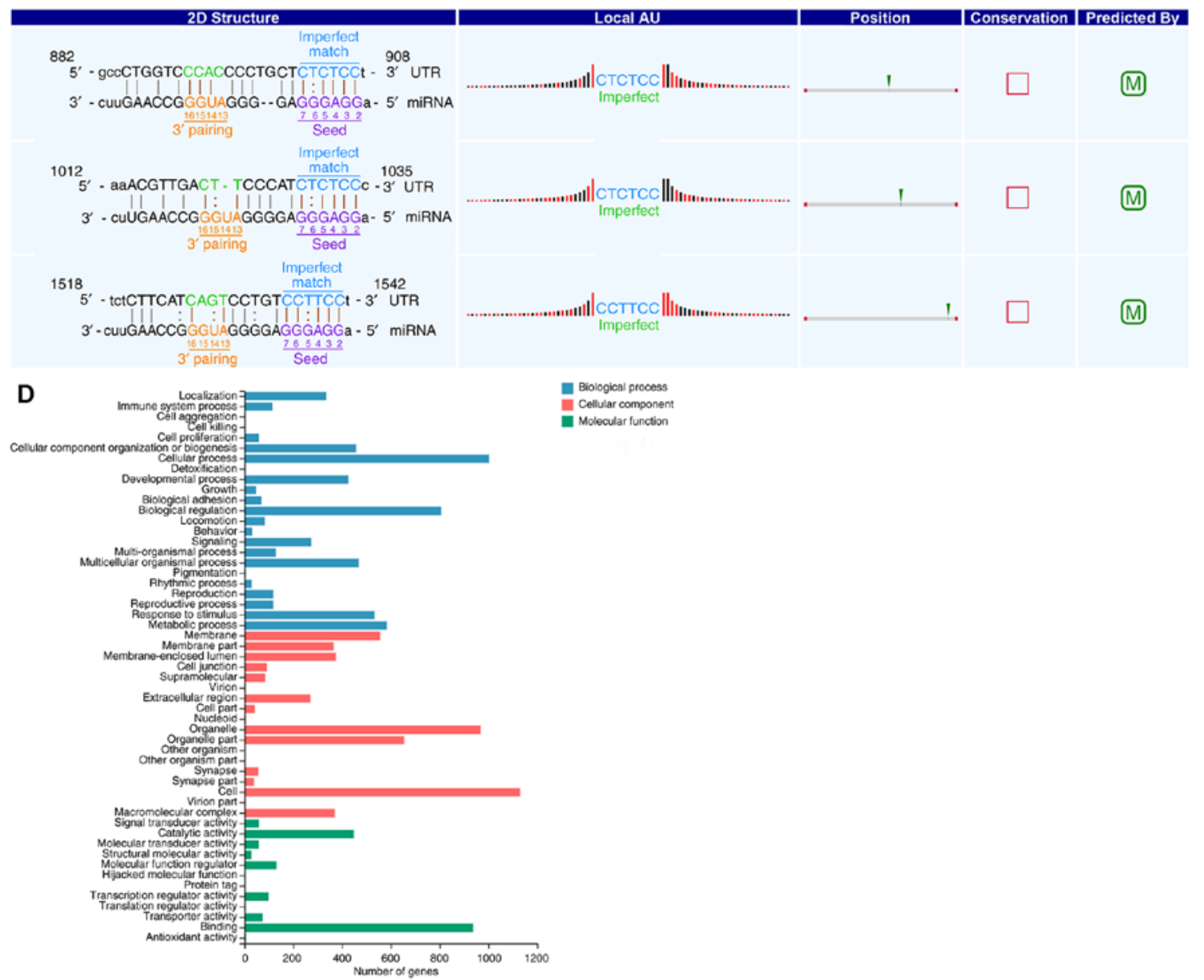

Figure 2. Microarray hybridization analysis (A) Cytoscape software was used to create a network map of mmu_circRNA_003795 with miRNAs that exhibited differential expression. (B) Cytoscape software was used to create a network map of mmu_circRNA_003795 with target miRNAs. (C) Potential binding sites of mmu_circRNA_003795 and miR-1249-5p were predicted using bioinformatics tools. (D) Annotation of the parental genes of the differentially expressed mRNAs using GO analysis. GO, Gene Ontology; circRNA, circular RNA; OPN, osteopontin; miRs/miRNAs, microRNAs; UTR, untranslated region.

This result was also demonstrated in MDPC23 cells (Fig. 4D). Western blotting revealed the same result. When miR1249-5p was upregulated or downregulated, the protein expression levels of COL15A1 and OPN were downregulated and upregulated, respectively (Fig. 4E and F). Compared with the negative control group, the staining and activity of ALP in the
mmu_circRNA_003795 inhibition group was significantly reduced at 7 days (Fig. 5A). In addition, the ARS staining at 21 days revealed the same result. Following osteogenic induction for 21 days, the intensity of ARS staining in the mmu_circRNA_003795 inhibition group was significantly reduced. Compared with the negative control group, the 
A

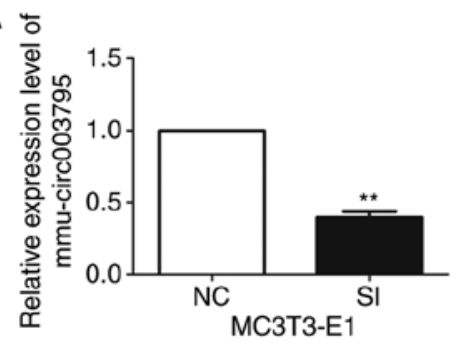

D

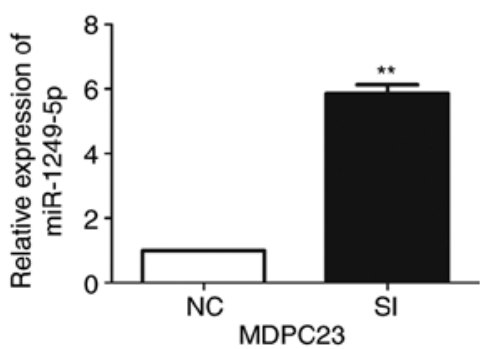

B

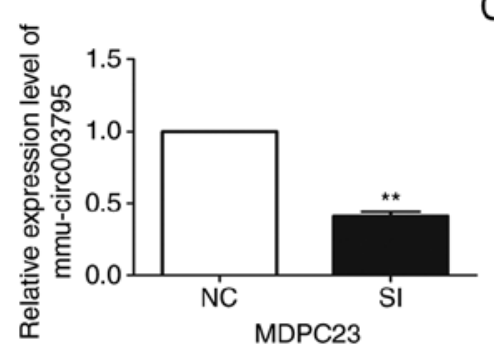

E

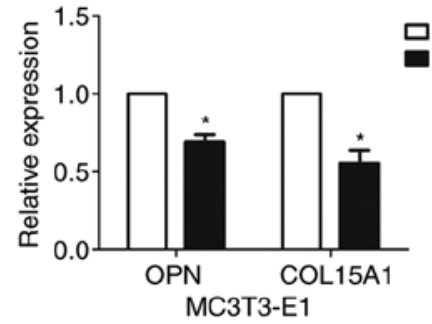

C

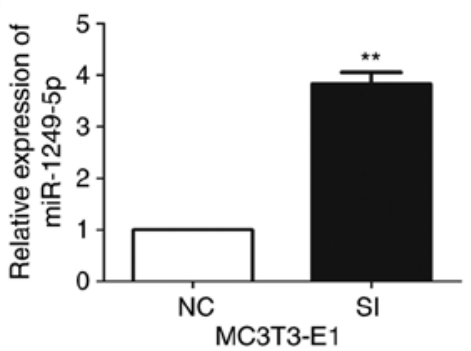

G

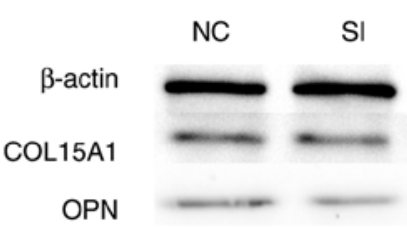

МСЗТЗ-E1

$\mathrm{H}$

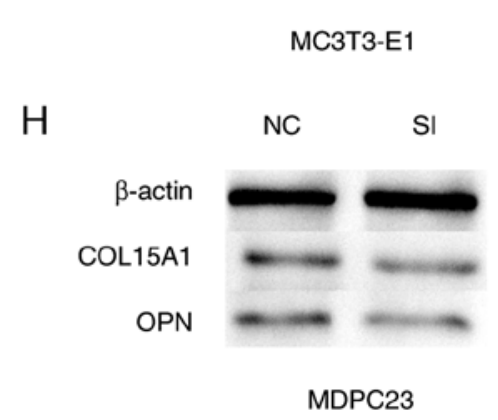

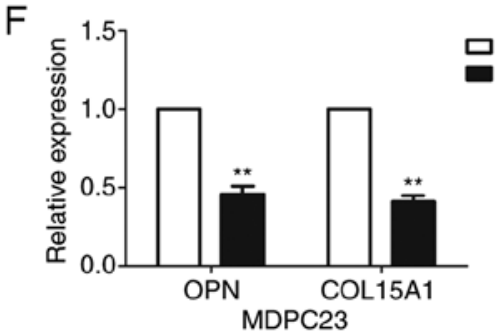
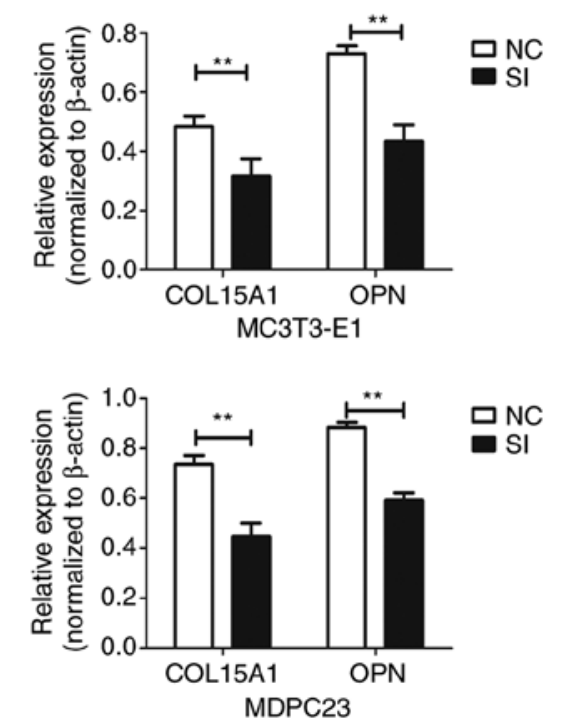

Figure 3. Changes of miR-1249-5p, COL15A1 and OPN expression following mmu_circRNA_003795 silencing. Expression of mmu_circRNA_003795 in the interference and NC groups, as analyzed by RT-qPCR in (A) MC3T3-E1 and (B) MDPC23 cells. Successful transfection and knockdown, and increased expression of miR-1249-5p in (C) MC3T3-E1 and (D) MDPC23 cells, and COL15A1 and OPN also in (E) MC3T3-E1 and (F) MDPC23 cells following mmu_circRNA_003795 silencing, as analyzed by RT-qPCR. Western blotting analysis of COL15A1 and OPN following mmu_circRNA_003795 silencing in (G) MC3T3-E1 and (H) MDPC23 cells. " $\mathrm{P}<0.05$ and ${ }^{* *} \mathrm{P}<0.01$ vs. NC. circRNA, circular RNA; OPN, osteopontin; RT-qPCR, reverse transcription-quantitative PCR; miR, microRNA; COL15A1, collagen type XV $\alpha 1$ chain; NC, negative control; SI, small inhibitor.

matrix mineralization of the mmu_circRNA_003795 inhibition group was decreased (Fig. 5B and C).

\section{Discussion}

circRNAs are circular RNA molecules that were first identified in the 1970s (15). Using electron microscopy, Hsu and Coca-Prados observed RNA could be a cyclic form in the cytoplasm of eukaryotic cells (16). With the continuous improvement of technology, circRNAs have become an increasingly important research area with huge development potential. circRNAs are now understood to exhibit structural stability, have conserved sequences and be extensively expressed in mammals $(17,18)$. Additionally, circRNAs are tissue and time specific. MDPC23 cells are mouse odontoblast cells derived from the oral dentin of mice, while MC3T3-E1 cells are derived from the bone of mice. The tissue specificity of the two may explain the differences in the osteogenic mineralization ability when mmu_circRNA_003795 was inhibited. However, this needs to be further investigated in future experiments.

COL15A1, a novel marker in osteoblasts, is associated with important biological functions in osteogenic differentiation and mineralization. Lisignoli et al (19) identified that COL15A1 is differentially expressed between osteoblasts and MSCs that were isolated from the same donors using high throughput technology. Trošt et al (20) isolated primary cultures of osteoblasts from osteoporotic and non-osteoporotic human bone tissue samples. Using genome-wide gene expression sequencing, this previous study found COL15A1 was downregulated in osteoporotic bone tissue compared with 
A
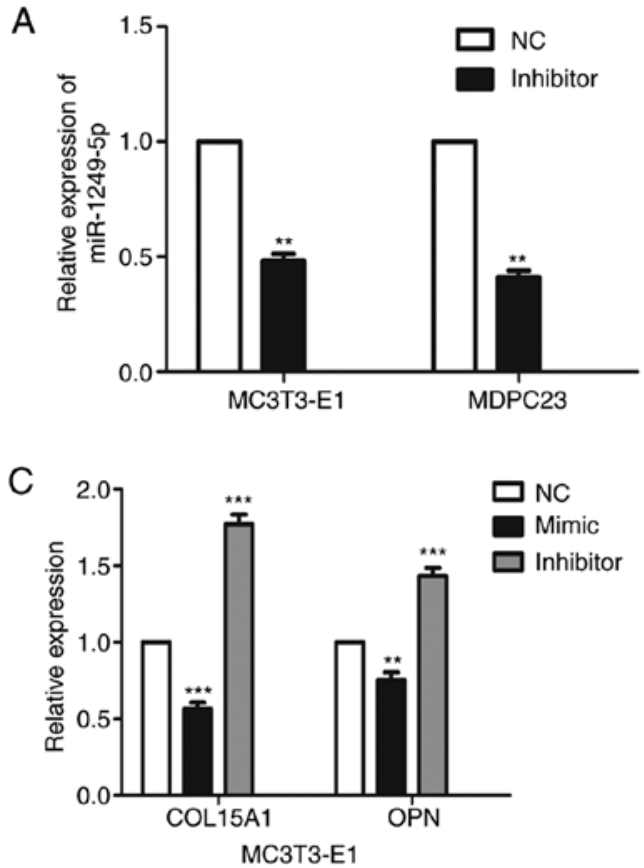

E

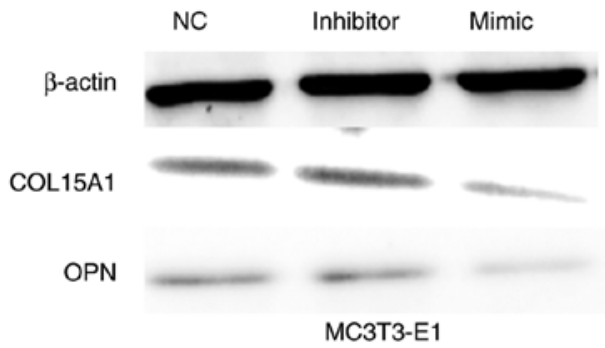

F

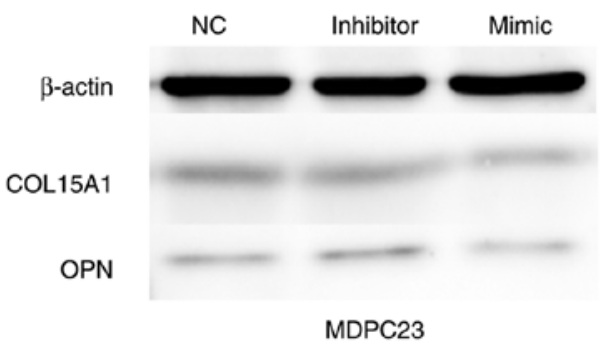

B

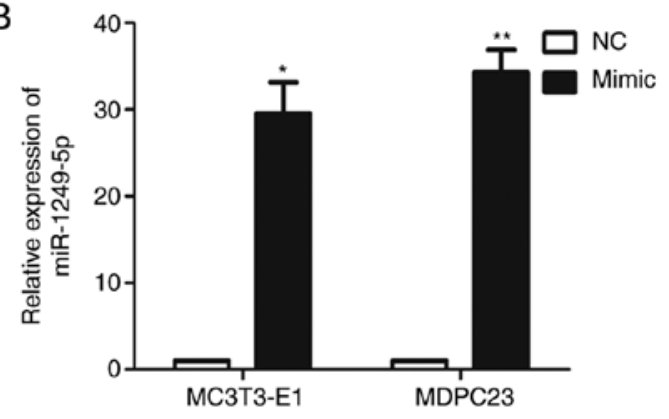

D
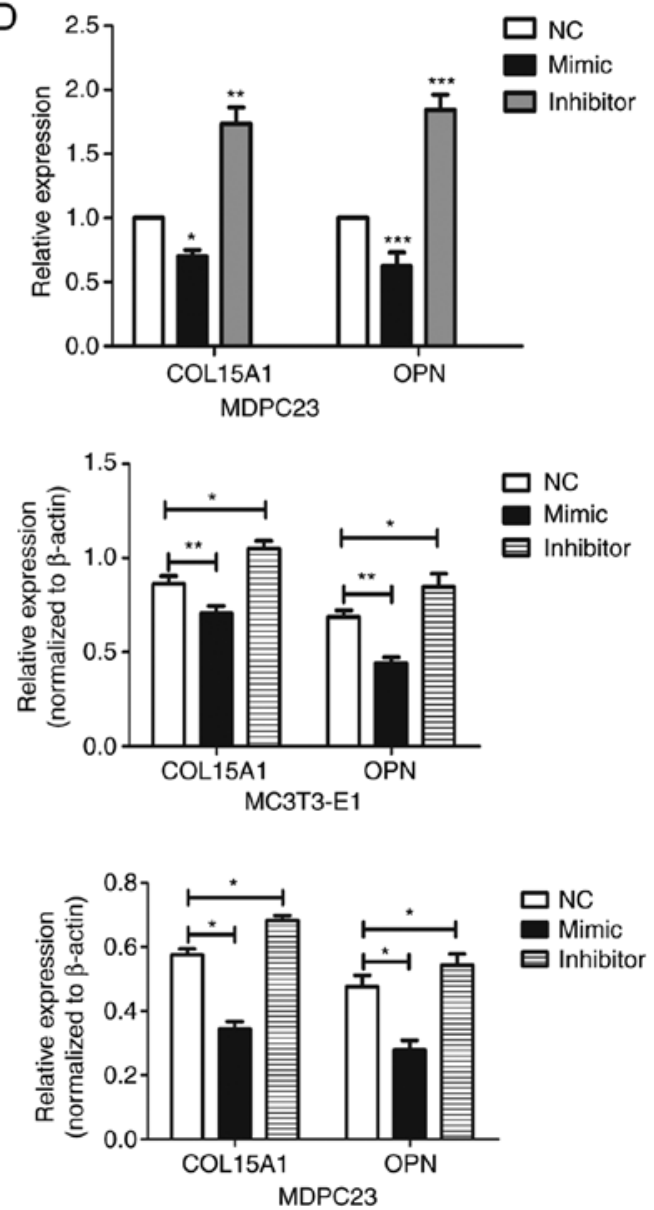

Figure 4. Silencing and overexpressing miR-1249-5p affects MC3T3-E1 and MDPC23 cells. RT-qPCR analysis of miR-1249-5p in MC3T3-E1 and MDPC23 cells following (A) silencing and (B) overexpressing miR-1249-5p. RT-qPCR analysis of COL15A1 and OPN in (C) MC3T3-E1 and (D) MDPC23 cells following silencing and overexpressing miR-1249-5p. Western blot analysis of COL15A1 and OPN following silencing and overexpressing miR-1249-5p in (E) MC3T3-E1 and (F) MDPC23 cells. "P<0.05, ${ }^{* *} \mathrm{P}<0.01$ and ${ }^{* * *} \mathrm{P}<0.005$. circRNA, circular RNA; OPN, osteopontin; RT-qPCR, reverse transcription-quantitative PCR; miR, microRNA; COL15A1, collagen type XV $\alpha 1$ chain.

non-osteoporotic human bone tissue. However, Gabusi et al reported that when chronically stimulated by $\mathrm{Ca}^{2+}$ at certain concentrations, the osteogenic ability of human osteoblasts was significantly enhanced, whereas the expression of COL15A1 was reduced (21).

OPN is a protein widely distributed in various tissues and cells, and it can participate in tissue repair, metabolism and other functions. OPN is associated with a variety of pathological processes, including cardiovascular disease, cancer, diabetes and kidney stones. OPN is also associated with physiological activities, such as cell viability, biomineralization and wound healing (22-25). OPN can regulate osteoclast function by influencing the expression levels of interleukin (IL)-10,
IL-12 and IL-3 (26). Mineralized tissues, such as tooth and bones, release OPN that is generated by osteoclasts and osteoblasts. Additionally, OPN can enhance the adhesion of osteoblasts, osteoclasts and bone cells (27). In the mineralized collagen matrix during the formation of bone tissue, the adhesion of bone cells is upregulated through concentrating OPN $(26,28)$. In the present study, MC3T3-E1 and MDPC23 cells were cultured in osteogenic induction medium containing siRNA. When the mineralization effect was tested by ALR staining after 21 days, compared with the control group, it was identified that the mineralized nodules in the 48-well plate were reduced, which may be due to the siRNA inhibiting the expression of COL15A1 and OPN, and ultimately affecting 
A

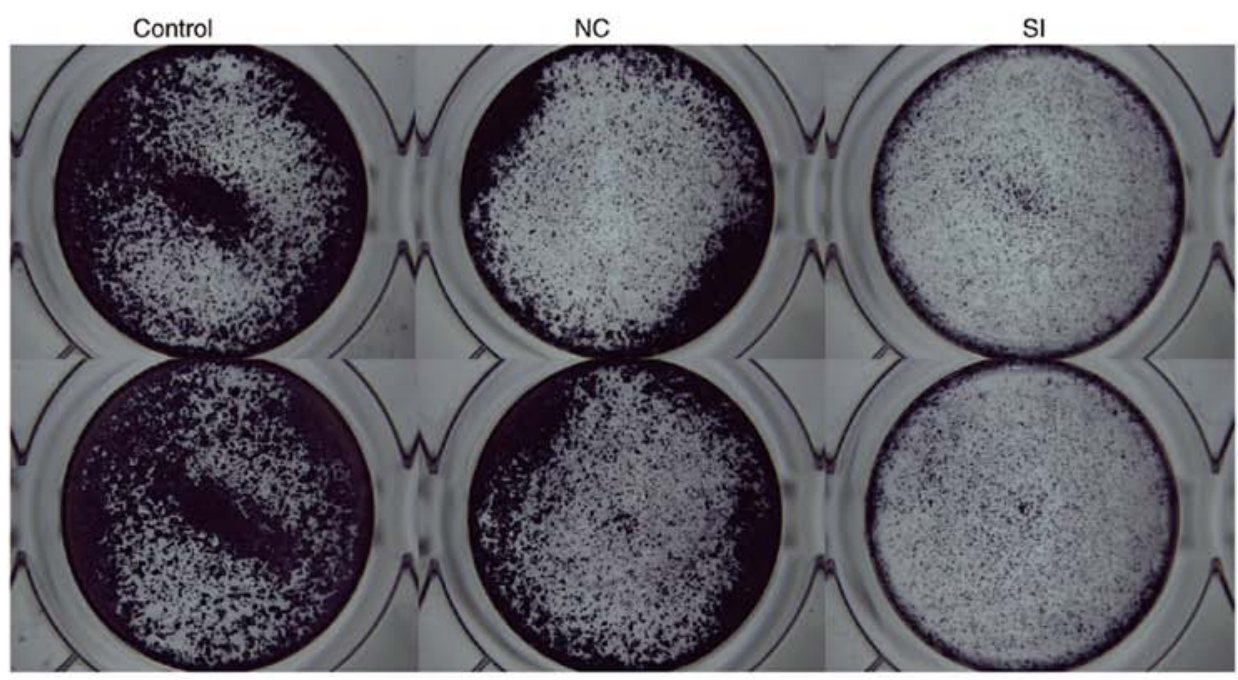

B

MDPC23

МСзтз-Е1
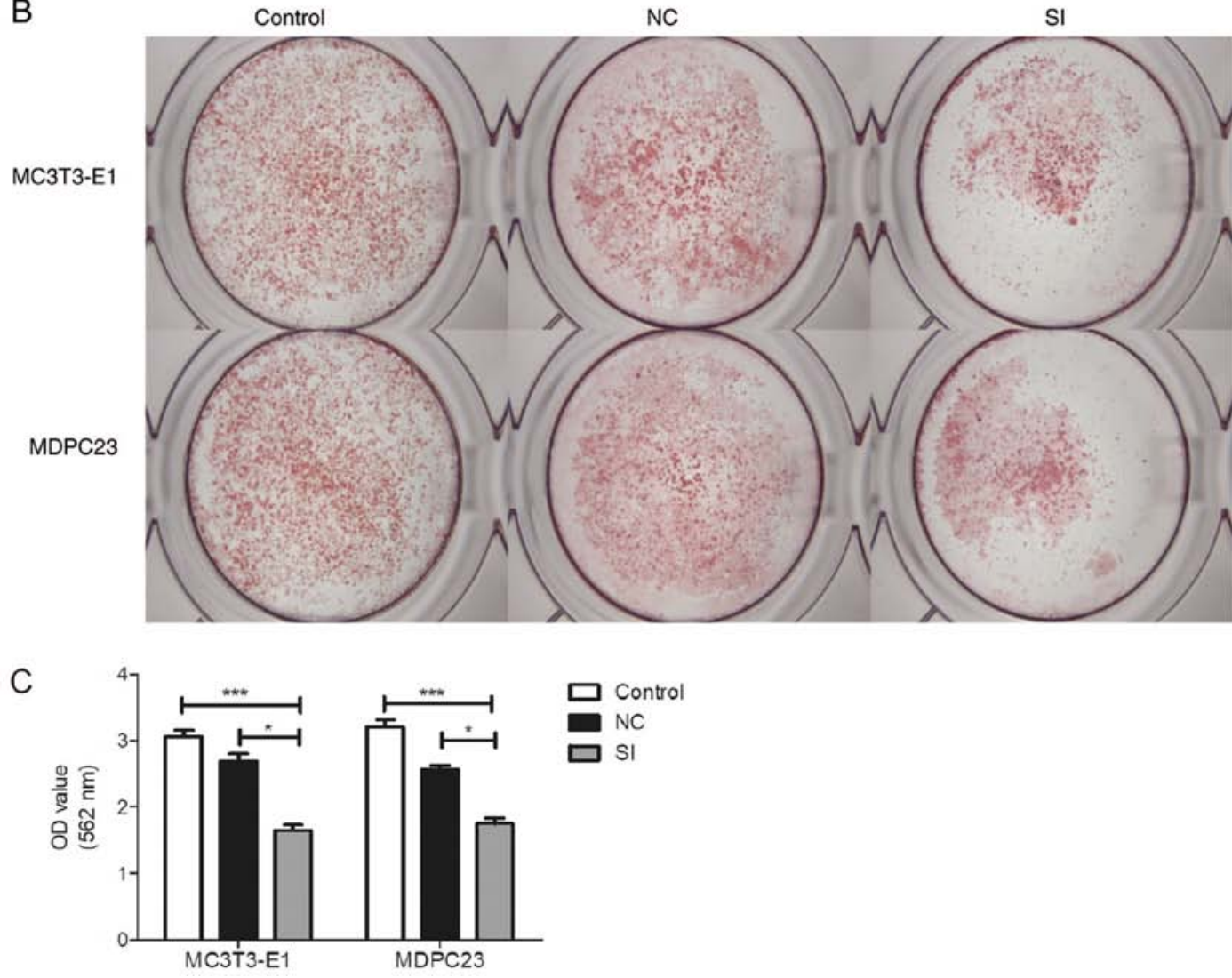

Figure 5. Silencing of mmu_circ_003795 affects osteogenesis of MC3T3-E1 and MDPC23 cells. (A) ALP assay of MC3T3-E1 and MDPC23 cells 7 days after siRNA transfection. (B) ARS assay of MC3T3-E1 and MDPC23 cells 21 days after siRNA transfection. (C) Calcium nodules quantification assay. ${ }^{*} \mathrm{P}<0.05$ and ${ }^{* * *} \mathrm{P}<0.005$. ARS, Alizarin Red-S; siRNA, small interfering RNA; miR, microRNA;circRNA, circular RNA; ALP, alkaline phosphatase, tissue nonspecific isozyme; OCN, osteocalcin; RUNX2, runt-related transcription factor 2; OSX, Sp7 transcription factor; COL15A1, collagen type XV $\alpha 1$ chain; OPN, osteopontin; NC, negative control.

the cell adhesion and osteogenesis. Due to their strong osteogenesis, ease of culture and availability, MC3T3-E1 and MDPC23 cells are considered good candidates for alveolar bone regeneration $(29,30)$. Therefore, it is important to understand the mechanism that regulates the differentiation of MDPC23 and MC3T3-E1 cells. circRNAs serve an important regulatory role in physiological activities (31). As a result of their abundant, stable and cell-specific expression, circRNAs are ideal biomarkers for the diagnosis of cancer, Alzheimer's disease, bone disease and other diseases (32-35). However, only a few studies have investigated the role of circRNAs during osteogenesis $(36,37)$. Recently, the expression of circRNAs in the MC3T3-E1 cell line during osteogenic differentiation was studied (7). The present study suggested that mmu_circ_003795 regulates the osteoblast differentiation and mineralization in MC3T3-E1 and MDPC23 cells. The current study identified the mRNAs that are associated with the osteoblast differentiation and mineralization of MDPC23 cells. 
The expression of corresponding parental genes can be increased by circRNAs through polymerase II elongation mechanism (17). Consequently, the present study investigated the regulatory role of mmu_circ_003795 by annotating the parental genes via GO analysis. The results revealed a large number of GO terms in the cellular processes and biological processes that were related to the osteogenic differentiation of cells. Previous studies have often focused on signaling proteins and osteogenic markers that play a key role in osteogenic differentiation $(38,39)$. For example, ALP, OCN and calcium deposition have been largely studied (40). Whereas, only a few studies have evaluated the expression profile of circRNAs in osteoblastic differentiation $(41,42)$. The present study suggested that mmu_circ_003795 may play an important role in the differentiation and mineralization of MC3T3-E1 and MDPC23 osteoblasts by targeting COL15A1. The mRNA expression levels of OPN and COL15A1 were decreased when siRNA was used to knockdown the expression of mmu_circRNA_003795. Therefore, the silencing of mmu_circRNA_003795 expression confirmed the association between mmu_circRNA_003795, mmu_miR_1249-5p, COL15A1 mRNA and OPN mRNA.

In conclusion, the preliminary observations of the present study demonstrated that mmu_circRNA_003795 can regulate osteoblast differentiation and mineralization in MC3T3-E1 and MDPC23 cells by targeting COL15A1 and OPN.

\section{Acknowledgements}

The authors would like to thank Dr Yan Yongyong (Key Laboratory of Oral Medicine, Guangzhou Institute of Oral Disease, Stomatology Hospital of Guangzhou Medical University, Guangzhou, Guangdong, P.R. China) for his great help in writing this paper.

\section{Funding}

The present study was supported by the Science \& Technology Bureau of Guangdong Province (grant nos. 2017A050501041 and 2018B050502012) and the National Key R\&D Program of China (grant no. 2018YFB1106903).

\section{Availability of data and materials}

The datasets used and/or analyzed during the current study are available from the corresponding author on reasonable request.

\section{Authors' contributions}

JW, WR, ZZ, ZH, TL, FL, ZS, QJ, LG and XY performed the experiments and analyzed the data. LG and $\mathrm{ZZ}$ designed the study and wrote the article. All authors approved the final submission.

\section{Ethics approval and consent to participate}

Not applicable.

\section{Patient consent for publication}

Not applicable.

\section{Competing interests}

The authors declare that they have no competing interests.

\section{References}

1. Bianco P, Robey PG and Simmons PJ: Mesenchymal stem cells: Revisiting history, concepts, and assays. Cell Stem Cell 2: 313-319, 2008.

2. Long T, Guo Z, Han L, Yuan X, Liu L, Jing W, Tian W, Zheng XH, Tang $\mathrm{W}$ and Long J: Differential expression profiles of circular RNÂs during osteogenic differentiation of mouse adipose-derived stromal cells. Calcif Tissue Int 103: 338-352, 2018.

3. Deng T, Yang L, Zheng Z, Li Y, Ren W, Wu C and Guo L: Calcitonin gene-related peptide induces IL-6 expression in RAW264.7 macrophages mediated by mmu_circRNA_007893. Mol Med Rep 16: 9367-9374, 2017.

4. Jeck WR, Sorrentino JA, Wang K, Slevin MK, Burd CE, Liu J, Marzluff WF and Sharpless NE: Circular RNAs are abundant, conserved, and associated with ALU repeats. RNA 19: 141-157, 2013.

5. Zhang W, Dong R, Shu D, Du J, Fan Z and Wang F: Differential long noncoding RNA/mRNA expression profiling and functional network analysis during osteogenic differentiation of human bone marrow mesenchymal stem cells. Stem Cell Res Ther 8: 30, 2017.

6. Li P, Chen S, Chen H, Mo X, Li T, Shao Y, Xiao B and Guo J: Using circular RNA as a novel type of biomarker in the screening of gastric cancer. Clin Chim Acta 444: 132-136, 2015.

7. Wu C, Zheng Z, Ren W, Deng T, Li Y, Yang L, Wu J, Huang Z, Li Z and Guo L: Mm9_circ_009056 enhances osteogenesis by targeting BMP7 via CGRP-mediated miR-22-3p. Biochem Biophys Res Commun 501: 199-205, 2018.

8. Li X, Zheng Y, Zheng Y, Huang Y, Zhang Y, Jia L and Li W: Circular RNA CDR1as regulates osteoblastic differentiation of periodontal ligament stem cells via the miR-7/GDF5/SMAD and p38 MAPK signaling pathway. Stem Cell Res Ther 9: 232, 2018.

9. Ren W, Yang L, Deng T, Wu C, Li Y, Wu J, Huang Z, Du F and Guo L: Calcitonin gene related peptide regulates FOSL2 expression and cell proliferation of BMSCs via mmu_circRNA_003795. Mol Med Rep 19: 3732-3742, 2019.

10. Chen K, Liu J, Liu S, Xia M, Zhang X, Han D, Jiang Y, Wang C and Cao X: Methyltransferase SETD2-mediated methylation of STAT1 is critical for interferon antiviral activity. Cell 170: 492-506.e14, 2017.

11. Livak KJ and Schmittgen TD: Analysis of relative gene expression data using real-time quantitative PCR and the 2(-Delta Delta C(T)) method. Methods 25: 402-408, 2001.

12. R Core Team: R: A language and environment for statistical computing. Version 3.1.2. R foundation for statistical computing, Vienna, 2014.

13. Grossmann S, Bauer S, Robinson PN and Vingron M: Improved detection of overrepresentation of gene-ontology annotations with parent child analysis. Bioinformatics 23: 3024-3031, 2007.

14. Standal T, Borset M and Sundan A: Role of osteopontin in adhesion, migration, cell survival and bone remodeling. Exp Oncol 26: 179-184, 2004.

15. Sanger HL, Klotz G, Riesner D, Gross HJ and Kleinschmidt AK: Viroids are single-stranded covalently closed circular RNA molecules existing as highly base-paired rod-like structures. Proc Natl Acad Sci USA 73: 3852-3856, 1976.

16. Hsu MT and Coca-Prados M: Electron microscopic evidence for the circular form of RNA in the cytoplasm of eukaryotic cells. Nature 280: 339-340, 1979.

17. Hansen TB, Jensen TI, Clausen BH, Bramsen JB, Finsen B, Damgaard CK and Kjems J: Natural RNA circles function as efficient microRNA sponges. Nature 495: 384-388, 2013.

18. Zhang Y, Zhang XO, Chen T, Xiang JF, Yin QF, Xing YH, Zhu S, Yang L and Chen LL: Circular intronic long noncoding RNAs. Mol Cell 51: 792-806, 2013.

19. Lisignoli G, Codeluppi K, Todoerti K, Manferdini C, Piacentini A, Zini N, Grassi F, Cattini L, Piva R, Rizzoli V, et al: Gene array profile identifies collagen type XV as a novel human osteoblast-secreted matrix protein. J Cell Physiol 220: 401-409, 2010.

20. Trošt Z, Trebše R, Preželj J, Komadina R, Logar DB and Marc J: A microarray based identification of osteoporosis-related genes in primary culture of human osteoblasts. Bone 46: 72-80, 2010. 
21. Gabusi E, Manferdini C, Grassi F, Piacentini A, Cattini L, Filardo G, Lambertini E, Piva R, Zini N, Facchini A and Lisignoli G: Extracellular calcium chronically induced human osteoblasts effects: Specific modulation of osteocalcin and collagen type XV. J Cell Physiol 227: 3151-3161, 2012.

22. Iaffaldano P, Ruggieri M, Viterbo RG, Mastrapasqua $M$ and Trojano M: The improvement of cognitive functions is associated with a decrease of plasma Osteopontin levels in Natalizumab treated relapsing multiple sclerosis. Brain Behav Immun 35: 176-181, 2014

23. Clemente N, Raineri D, Cappellano G, Boggio E, Favero F, Soluri MF, Dianzani C, Comi C, Dianzani U and Chiocchetti A: Osteopontin bridging innate and adaptive immunity in autoimmune diseases. J Immunol Res 2016: 7675437, 2016.

24. Standal T, Borset M and Sundan A: Role of osteopontin in adhesion, migration, cell survival and bone remodeling. Exp Oncol 26: 179-184, 2004.

25. Coppola D, Szabo M, Boulware D, Muraca P, Alsarraj M, Chambers AF and Yeatman TJ: Correlation of osteopontin protein expression and pathological stage across a wide variety of tumor histologies. Clin Cancer Res 10: 184-190, 2004.

26. Nau GJ, Liaw L, Chupp GL, Berman JS, Hogan BL and Young RA: Attenuated host resistance against Mycobacterium bovis BCG infection in mice lacking osteopontin. Infect Immun 67: 4223-4230, 1999.

27. Reinholt FP, Hultenby K, Oldberg A and Heinegård D: Osteopontin-a possible anchor of osteoclasts to bone. Proc Natl Acad Sci USA 87: 4473-4475, 1990.

28. Lenton S, Seydel T, Nylander T, Holt C, Härtlein M, Teixeira S and Zaccai G: Dynamic footprint of sequestration in the molecular fluctuations of osteopontin. J R Soc Interf 12: 506, 2015.

29. Schwarz F, Golubovic V, Becker K and Mihatovic I: Extracted tooth roots used for lateral alveolar ridge augmentation: A proof-of-concept study. J Clin Periodontol 43: 345-353, 2016.

30. Schwarz F, Schmucker A and Becker J: Initial case report of an extracted tooth root used for lateral alveolar ridge augmentation. J Clin Periodontol 43: 985-989, 2016.

31. Liu J, Liu T, Wang $X$ and He A: Circles reshaping the RNA world: From waste to treasure. Mol Cancer 16: 58, 2017.

32. Hansen TB, Kjems J and Damgaard CK: Circular RNA and miR-7 in cancer. Cancer Res 73: 5609-5612, 2013.
33. Wang F, Nazarali AJ and Ji S: Circular RNAs as potential biomarkers for cancer diagnosis and therapy. Am J Cancer Res 6: 1167-1176, 2016.

34. Peng L, Yuan XQ and Li GC: The emerging landscape of circular RNA ciRS-7 in cancer (Review). Oncol Rep 33: 2669-2674, 2015.

35. Liu Q, Zhang X, Hu X, Dai L, Fu X, Zhang J and Ao Y: Circular RNA related to the chondrocyte ECM regulates MMP13 expression by functioning as a MiR-136 'Sponge' in human cartilage degradation. Sci Rep 6: 22572, 2016.

36. Zheng Y, Li X, Huang Y, Jia L and Li W: The circular RNA landscape of periodontal ligament stem cells during osteogenesis. J Periodontol 88: 906-914, 2017.

37. Huang X, Cen X, Zhang B, Liao Y, Zhu G, Liu J and Zhao Z: Prospect of circular RNA in osteogenesis: A novel orchestrator of signaling pathways. J Cell Physiol 234: 21450-21459, 2019.

38. Yu X, Li Z, Wan Q, Cheng X, Zhang J, Pathak JL and Li Z: Inhibition of JAK2/STAT3 signaling suppresses bone marrow stromal cells proliferation and osteogenic differentiation, and impairs bone defect healing. Biol Chem 399: 1313-1323, 2018.

39. Lee SH, Park Y, Song M, Srikanth S, Kim S, Kang MK, Gwack Y, Park NH, Kim RH and Shin KH: Orail mediates osteogenic differentiation via BMP signaling pathway in bone marrow mesenchymal stem cells. Biochem Biophys Res Commun 473: 1309-1314, 2016

40. Cotter EJ, Ip HS, Powderly WG and Doran PP: Mechanism of HIV protein induced modulation of mesenchymal stem cell osteogenic differentiation. BMC Musculoskelet Disord 9: 33, 2008.

41. Li X, Zheng Y, Zheng Y, Huang Y, Zhang Y, Jia L and Li W: Circular RNA CDR1as regulates osteoblastic differentiation of periodontal ligament stem cells via the miR-7/GDF5/SMAD and p38 MAPK signaling pathway. Stem Cell Res Ther 9: 232, 2018.

42. Qian DY, Yan GB, Bai B, Chen Y, Zhang SJ, Yao YC and Xia H: Differential circRNA expression profiles during the BMP2-induced osteogenic differentiation of MC3T3-E1 cells. Biomed Pharmacother 90: 492-499, 2017.

This work is licensed under a Creative Commons Attribution-NonCommercial-NoDerivatives 4.0 International (CC BY-NC-ND 4.0) License. 